Article

\title{
Identification of Novel Thiazolo[5,4-b]Pyridine Derivatives as Potent Phosphoinositide 3-Kinase Inhibitors
}

\author{
Liang Xia ${ }^{1,2}$, Yan Zhang ${ }^{1,2}$, Jingbo Zhang ${ }^{1,2}$, Songwen Lin ${ }^{1,2}$, Kehui Zhang ${ }^{1,2}$, Hua Tian ${ }^{1,2}$, \\ Yi Dong ${ }^{1,2, *}$ and Heng $X u^{1,2, *}$ \\ 1 State Key Laboratory of Bioactive Substance and Function of Natural Medicines, Institute of Materia Medica, \\ Chinese Academy of Medical Sciences and Peking Union Medical College, Beijing 100050, China; \\ xialiang@imm.ac.cn (L.X.); zhangyan@imm.ac.cn (Y.Z.); zhangjingbo@imm.ac.cn (J.Z.); \\ linsongwen@imm.ac.cn (S.L.); kehuizhang@imm.ac.cn (K.Z.); tianh@imm.ac.cn (H.T.) \\ 2 Beijing Key Laboratory of Active Substances Discovery and Druggability Evaluation, Institute of Materia \\ Medica, Chinese Academy of Medical Sciences and Peking Union Medical College, Beijing 100050, China \\ * Correspondence: dongyi@imm.ac.cn (Y.D.); xuheng@imm.ac.cn (H.X.)
}

Academic Editors: Panayiotis A. Koutentis and Andreas S. Kalogirou

Received: 10 September 2020; Accepted: 1 October 2020; Published: 12 October 2020

check for updates

\begin{abstract}
A series of novel 2-pyridyl, 4-morpholinyl substituted thiazolo[5,4- $b$ ]pyridine analogues have been designed and synthesized in this paper. These thiazolo[5,4- $b]$ pyridines were efficiently prepared in seven steps from commercially available substances in moderate to good yields. All of these $N$-heterocyclic compounds were characterized by nuclear magnetic resonance (NMR) and high-resolution mass spectrometry (HRMS) analysis and tested for phosphoinositide 3-kinase (PI3K) enzymatic assay. The results indicated that these $N$-heterocyclic compounds showed potent PI3K inhibitory activity, and the $\mathrm{IC}_{50}$ of a representative compound (19a) could reach to $3.6 \mathrm{~nm}$. The structure-activity relationships (SAR) study showed that sulfonamide functionality was important for $\mathrm{PI} 3 \mathrm{~K} \alpha$ inhibitory activity, and 2-chloro-4-florophenyl sulfonamide (19b), or 5-chlorothiophene-2-sulfonamide (19c) showed potent inhibitory activity with a nanomolar $\mathrm{IC}_{50}$ value. The pyridyl attached to thiazolo[5,4- $\left.b\right]$ pyridine was another key structural unit for $\mathrm{PI} 3 \mathrm{~K} \alpha$ inhibitory potency, and replacement by phenyl lead to a significant decrease in activity. Enzymatic Inhibition results showed that compound 19a inhibited PI3K $\alpha, \mathrm{PI} 3 \mathrm{~K} \gamma$, or PI3K $\delta$ with a nanomolar $\mathrm{IC}_{50}$ value, but its inhibitory activity on PI3K $\beta$ was approximately 10 -fold reduced. Further docking analysis revealed that the $N$-heterocyclic core of compound 19a was directly involved in the binding to the kinase through the key hydrogen bonds interaction.
\end{abstract}

Keywords: heterocycle; thiazolo[5,4-b]pyridine; phosphoinositide 3-kinase; inhibitory potency; docking analysis

\section{Introduction}

Heterocycles are important organic chemical structures commonly found in a large number of agrochemicals, materials, and pharmaceutical molecules [1-3]. Heterocyclic structural units are especially prevalent in synthetic drug molecules because they can improve the interaction between drug molecules and proteins and regulate the physicochemical properties of drugs [4-6]. It is estimated that about $70 \%$ of pharmaceutical products contain heterocyclic substructures, which clearly shows that heterocycles play an extremely important role in drug development and discovery $[7,8]$.

Over the past few decades, many biological compounds containing bicyclic heterocycles have been found with potent pharmacological activities; moreover, some of them have also been successfully developed into drugs approved for marketing $[9,10]$. As a typical [5,6]-fused bicyclic scaffold, 
thiazolo[5,4- $b]$ pyridine has been regarded as an important privileged structure in medicinal chemistry because of its structural similarity to thiazolo[4,5- $d$ ]pyrimidine, which is a classic biologically useful skeleton [11]. Furthermore, thiazolo[5,4-b]pyridine analogues have been found to exhibit a range of biological activities, such as S1p1 and S1p5 agonist (1) [12], $\mathrm{H}_{3}$ receptor antagonist (2) [13], DNA gyrase B inhibitor (3) [14], anticancer agent (4) [15], and glucokinase activator (5) [16], among others (Figure 1). A few of them show promising drug-like properties, which has led to increased interest in the design, synthesis, and bioactivity evaluation of thiazolo[5,4- $b$ ]pyridine analogues. In this paper, the novel 2-pyridyl, 4-morpholinyl substituted thiazolo[5,4-b]pyridine has been designed and utilized as a template to afford multi-heterocyclic phosphoinositide 3-kinase (PI3K) inhibitors with high potency.

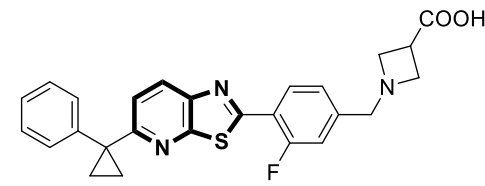

$1, \mathrm{~S}_{1} \mathrm{P}_{1}$ and $\mathrm{S}_{1} \mathrm{P}_{5}$ agonist

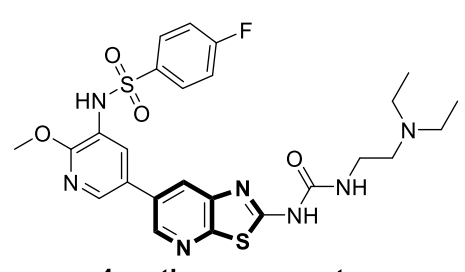

4, anticancer agent

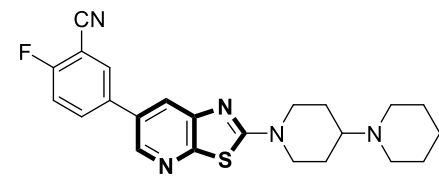

$2, \mathrm{H}_{3}$ receptor antagonists

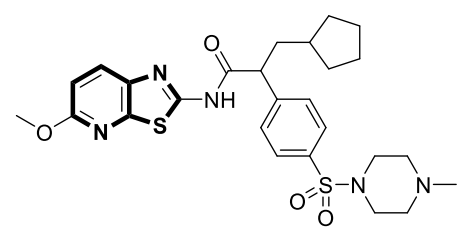

5 , glucokinase activators

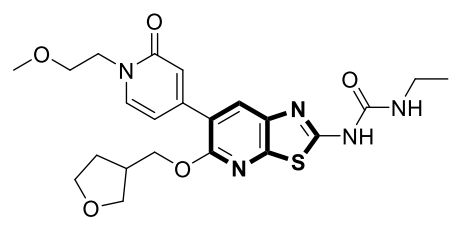

3, DNA gyrase B inhibitor

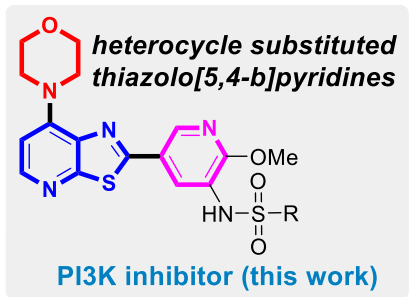

Figure 1. Selected biological compounds with thiazolo[5,4-b]pyridine skeleton.

The PI3Ks signaling pathway plays a crucial biological function in the process of cell growth, survival, proliferation, and differentiation, which has been proven to be an important target for tumor-targeted therapy [17]. In many tumor cells, the amplification, overexpression, or activating mutation of $\mathrm{PI} 3 \mathrm{~K} \alpha$ can lead to abnormal activation of the PI3K signaling pathway, which is a key driving force for tumor occurrence. Given that the PI3K signaling pathway is closely related to tumorigenesis, chemotherapy by inhibiting the PI3K signaling pathway has become an important means of tumor treatment [18], and some potent PI3K inhibitors have been discovered in recent years. It is worth noting that heteroaryl morpholines are common structural cores of PI3K inhibitor molecules-such as PI-103 (6) [19], BKM120 (7) [20], and GDC-0980 (8) [21]—and are representative PI3K inhibitors for the treatment for cancer. Another important core is the 2-methoxyl pyridine unit, which has been seen in PF-04691502 (9) [22] and GSK2126458 (10) [23] as PI3K/mTOR dual inhibitors. In this paper, we designed a new structural template consisting of morpholinyl and 2-methoxyl pyridine to obtain a series of thiazolo[5,4- $b$ ]pyridines with high PI3K inhibitory activity. Further analysis of the isoform inhibitory selectivity showed that compound 19a showed highly potent inhibition of PI3k $\alpha, \operatorname{PI} 3 k \gamma$, and $\mathrm{PI} 3 \mathrm{k} \delta$ with an $\mathrm{IC}_{50}$ of $3.4 \mathrm{nM}, 1.8 \mathrm{nM}$, and $2.5 \mathrm{nM}$, respectively. Furthermore, the $\mathrm{IC}_{50}$ value of PI3k $\beta$ was approximately 10-fold higher than the other three (Figure 2). 


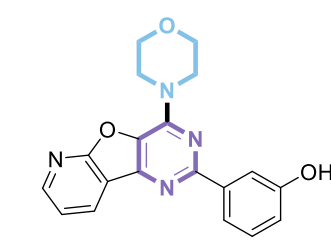

6 (PI-103)

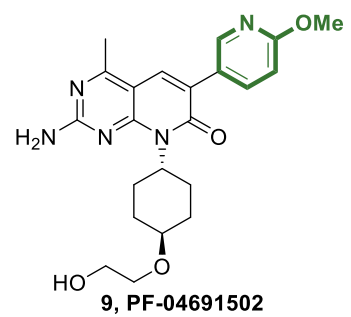

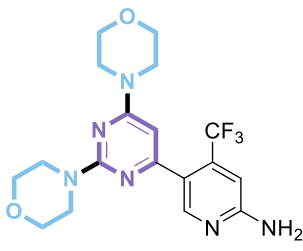

7 (BKM120)

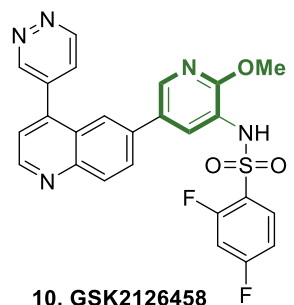

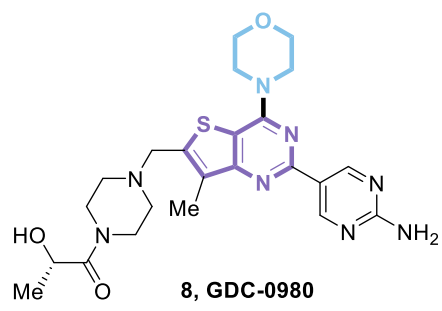

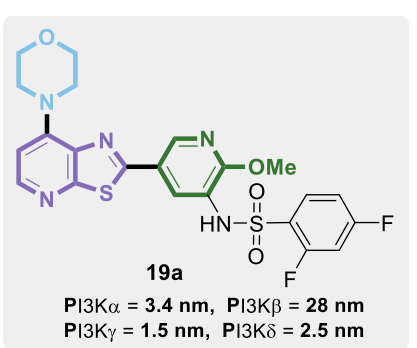

Figure 2. Design of 2-pyridyl, 4-morpholinyl substituted thiazolo[5,4-b]pyridine PI3K inhibitor.

\section{Results}

\subsection{Synthesis}

An effective and economical method for preparing the thiazolo[5,4-b]pyridine analogues is shown in Figure 3. A commercially available and inexpensive 2,4-dichloro-3-nitropyridine (11) was used as a starting material, and the designed seven-step synthetic route was simple and easy to handle. 2,4-dichloro-3-nitropyridine (11) could be smoothly transformed into 4-morpholinyl pyridine derivative 12 via selective substation with morpholine in the presence of triethylamine. Molecule 12 underwent another substitution with a thiocyanate by treatment with $\mathrm{KSCN}$ in acetic acid at $80{ }^{\circ} \mathrm{C}$ to successfully afford 4-(3-nitro-2-thiocyanatopyridin-4-yl)morpholine (13). The nitro group was reduced by treatment with Fe powder in acetic acid at $60{ }^{\circ} \mathrm{C}$; the subsequent intramolecular cyclization occurred in one-pot to construct the thiazolo[5,4- $b$ ]pyridine skeleton, and the corresponding amino thiazolo[5,4- $b]$ pyridine derivative (14) was obtained in moderate yield. A copper bromide mediated bromination effectively gave the bromothiazolo[5,4- $b$ ]pyridine derivative (15) at room temperature, which underwent a Suzuki reaction with aryl borates at $100{ }^{\circ} \mathrm{C}$ to afford the desired heterocycle substituted thiazolo $[5,4-b]$ pyridine analogues (19a-19f) in good yields. Sulfonamide substituted aryl borates were prepared from the corresponding amino-substituted aryl bromide through sulfonamidation and boronization in two steps.

\subsection{Enzymatic Assay}

All of the $N$-heterocycle substituted thiazolo[5,4-b]pyridine analogues were evaluated for their PI3K $\alpha$ inhibitory activity through enzymatic assay. The structure-activity relationships (SAR) are summarized in Table 1 . We firstly designed and synthesized an $N$-heterocyclic compound (19a) composed of methoxypyridine and morpholinyl thiazolo[5,4- $b$ ]pyridine. As expected, this thiazolo[5,4- $b$ ]pyridine compound exhibited extremely strong PI3k $\alpha$ inhibitory activity with an $\mathrm{IC}_{50}$ of $3.6 \mathrm{nM}$. In further SAR studies, the sulfonamide functionality was proved to be a key structural unit that affected activity. For example, despite a 2-3 fold drop in potency compared with that of 2,4-difluorophenyl sulfonamide, 2-chloro-4-florophenyl sulfonamide (19b), or 5-chlorothiophene-2-sulfonamide (19c) showed high inhibitory activity with an $\mathrm{IC}_{50}$ of $4.6 \mathrm{nM}$ and $8.0 \mathrm{nM}$, respectively. This activity was attributed to the fact that the electron-deficient aryl group resulted in a more acidic sulfonamide NH proton being able to make a stronger charged interaction with Lys802 in PI3K $\alpha$ [24]. Consistent with this finding, when 2,4-difluorophenyl was replaced with methyl (19d), the PI3K $\alpha$ inhibitory potency dropped over 10-fold $\left(\mathrm{IC}_{50}=53 \mathrm{nM}\right)$. Removal of the methoxyl group attached to the pyridine ring lead did not significantly affect the potency $\left(\mathrm{IC}_{50}=4.0 \mathrm{nM}\right)$. Replacement of pyridyl with phenyl resulted in an apparent decrease in PI3K $\alpha$ inhibitory activity 
$\left(\mathrm{IC}_{50}=501 \mathrm{nM}\right)$, demonstrating that the pyridyl attached to thiazolo[5,4-b]pyridine was a necessary moiety for enzymatic potency. We also evaluated the inhibitory selectivity of compound 19a against four isoforms of class I PI3K (Table 2). It potently inhibited PI3K $\alpha, \mathrm{PI} 3 \mathrm{~K} \gamma$, and PI3K $\delta$ with nanomolar $\mathrm{IC}_{50}$ values, which is approximately 10 -fold higher than that of $\mathrm{PI} 3 \mathrm{~K} \beta$, clearly proving that it is a novel and potent PI3K inhibitor.

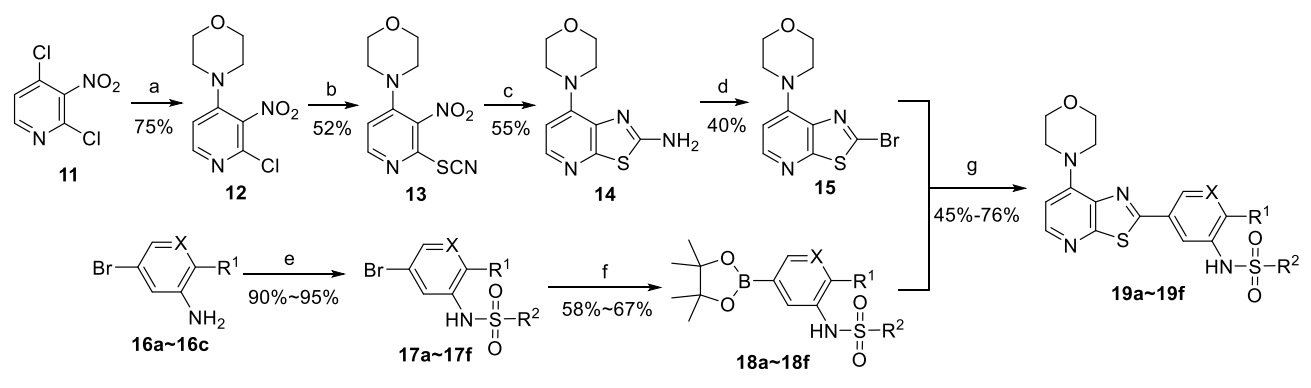

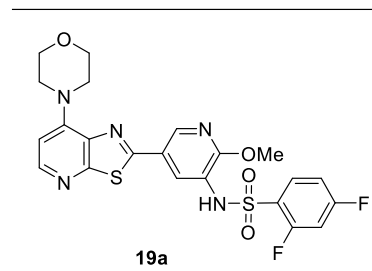

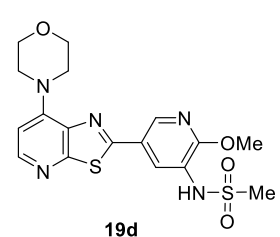

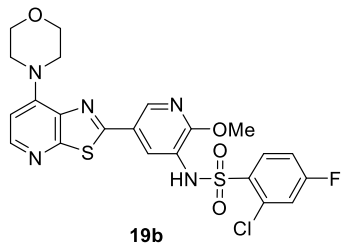

$19 b$

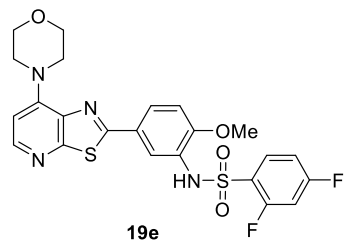

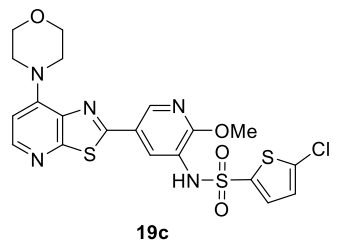

$19 c$

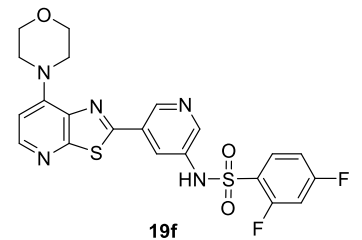

Figure 3. Synthesis of thiazolo[5,4-b]pyridine analogues. (a) Morpholine, TEA, THF, $0{ }^{\circ} \mathrm{C}$. (b) KSCN, $\mathrm{HOAc}, 80^{\circ} \mathrm{C}$. (c) Fe powder, $\mathrm{HOAc}, 60^{\circ} \mathrm{C}$. (d) $\mathrm{CuBr}_{2}$, tert-butyl nitrite, $\mathrm{CH}_{3} \mathrm{CN}$, rt. (e) DMAP, pyridine, $\mathrm{DCM}$, rt. (f) $\mathrm{PdCl}_{2}$ (dppf), $\mathrm{KOAc}$, bis(pinacolato)diboron, 1,4-dioxane, $100{ }^{\circ} \mathrm{C}$. (g) $\mathrm{PdCl}_{2}(\mathrm{dppf}), \mathrm{K}_{2} \mathrm{CO}_{3}$, 1,4-dioxane $/ \mathrm{H}_{2} \mathrm{O}$, aryl borate, $100{ }^{\circ} \mathrm{C}$. dppf $=1,1^{\prime}$-Bis(diphenylphosphino)ferrocene.

Table 1. SAR studies of 2-substitutions on the thiazolo[5,4- $b]$ pyridine.

\begin{tabular}{|c|c|c|c|c|c|c|}
\hline Compd & $x$ & $\mathbf{R}^{1}$ & $\mathbf{R}^{2}$ & $\operatorname{cLog}^{a}$ & PSA $^{a}$ & $\operatorname{PI} 3 K \alpha(n m)^{b}$ \\
\hline $19 a$ & $\mathrm{~N}$ & $\mathrm{OMe}$ & & 4.1 & 105.0 & 3.6 \\
\hline $19 b$ & $\mathrm{~N}$ & $\mathrm{OMe}$ & & 4.4 & 105.0 & 4.6 \\
\hline $19 \mathrm{c}$ & $\mathrm{N}$ & $\mathrm{OMe}$ & & 4.3 & 105.0 & 8.0 \\
\hline $19 d$ & $\mathrm{~N}$ & $\mathrm{OMe}$ & $M$ & 2.1 & 105.0 & 53 \\
\hline $19 e$ & $\mathrm{CH}$ & $\mathrm{OMe}$ & & 4.4 & 92.6 & 501 \\
\hline $19 f$ & $\mathrm{~N}$ & $\mathrm{H}$ & & 3.3 & 95.7 & 4.0 \\
\hline
\end{tabular}

$\overline{{ }^{a} \text { Calculated from ChemBioDraw Ultra 14.0. }{ }^{b} \text { Mean of at least three separate experiments. }}$ 
Table 2. Enzymatic inhibition by compound 19a ${ }^{a}$.

\begin{tabular}{cc}
\hline Enzymatic Assay & IC $_{\mathbf{5 0}}(\mathbf{n M})$ \\
\hline PI3K $\alpha$ & 3.6 \\
PI3K $\beta$ & 34 \\
PI3K $\gamma$ & 1.6 \\
PI3K $\delta$ & 2.9 \\
\hline
\end{tabular}

${ }^{a}$ Mean of at least three separate experiments.

\subsection{Molecular Docking Study}

Because of its superior PI3K potency, thiazolo[5,4-b]pyridine 19a was selected for a molecular docking study. As shown in Figure 4, the 2-pyridyl thiazolo[5,4-b]pyridine scaffold fits well into the ATP binding pocket of the PI3K $\alpha$ kinase, forming a hydrogen bond interaction with the Val851 residue of the hinge region and a water bridge with Typ836 and Asp810. It is worth noting that another hydrogen bond interaction was observed between the sulfonamide group and Lys802. These interaction networks are the key factors for the PI3K bonding of these pyridyl thiazolo[5,4-b]pyridine analogues.

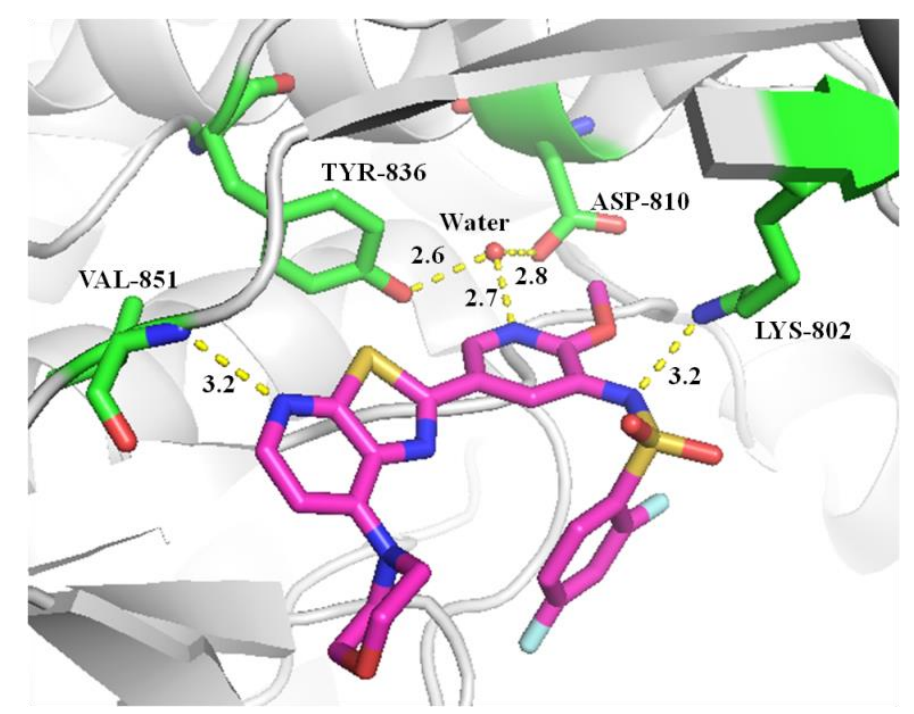

Figure 4. Predicted binding mode of 19a with PI3K $\alpha$.

\section{Materials and Methods}

\subsection{General}

Acetic acid, 1,4-dioxane, acetonitrile and dried THF were purchased from domestic corporations and used without further purification. Aryl borates were synthesized according to our previous work [24]. Nuclear magnetic resonance spectroscopy $\left({ }^{1} \mathrm{H}\right.$ NMR and ${ }^{13} \mathrm{C}$ NMR) was performed on Bruker Advance 400M NMR spectrometers, and high-resolution LC-MS was carried out by Agilent LC/MSD TOF. Purity of all compounds tested for PI3K activity were determined to be $>95 \%$ by LCMS analysis. The kinase inhibitory activity assay was performed by Shanghai ChemPartner Co., Ltd. (Shanghai, China). For details, see our previously published protocols [24]. Molecular docking study was conducted in the Schrodinger software.

\subsection{Synthesis}

\subsubsection{Synthesis of 4-(2-chloro-3-nitropyridin-4-yl)morpholine (12)}

Morpholine ( $50 \mathrm{mmol}, 4.36 \mathrm{~mL}$ ) was added dropwise to the mixture of 2,4-dichloro-3-nitropyridine $(\mathbf{1 1}, 50 \mathrm{mmol}, 9.6 \mathrm{~g})$ and trimethylamine $(50 \mathrm{mmol}, 7.0 \mathrm{~mL})$ in $\mathrm{THF}(100 \mathrm{~mL})$ at $0{ }^{\circ} \mathrm{C}$, then the reaction 
mixture was stirred at $0{ }^{\circ} \mathrm{C}$ for $1 \mathrm{~h}$, and the reaction was monitored by LC-MS. When the starting material was consumed completely, solvent was removed under reduced pressure, and the residue was purified with flash column chromatography on silica gel to afford compound 7 (9.1 g, $75 \%$ yield). Yellow solid, $\mathrm{R}_{\mathrm{f}}$ : 0.5 (EtOAc/Petroleum ether = 1:1), m.p.: $138-140{ }^{\circ} \mathrm{C} .{ }^{1} \mathrm{H}$ NMR (400 MHz, DMSO-d6) $\delta 8.22(\mathrm{~d}, J=6.0 \mathrm{~Hz}, 1 \mathrm{H}), 7.25(\mathrm{~d}, J=6.1 \mathrm{~Hz}, 1 \mathrm{H}), 3.69-3.64(\mathrm{~m}, 4 \mathrm{H}), 3.25-3.20(\mathrm{~m}, 4 \mathrm{H}) .{ }^{13} \mathrm{C}$ NMR (101 MHz, DMSO-d6) $\delta 149.9,149.7,142.3,128.0,113.9,65.5,48.6$. HRMS (ESI): $\mathrm{m} / z\left(\mathrm{M}+\mathrm{H}^{+}\right)$calcd for $\mathrm{C}_{9} \mathrm{H}_{11} \mathrm{ClN}_{3} \mathrm{O}_{3}, 244.0483$, found: 244.0492. IR (ATR): 3155, 2965, 2865, 1524, 1356, 1215, 1117, 964, 848, $719 \mathrm{~cm}^{-1}$. The data is consistent with the data reported in the literature. [25]

\subsubsection{Synthesis of 4-(3-nitro-2-thiocyanatopyridin-4-yl)morpholine (13)}

A mixture of KSCN (48 mmol, $4.7 \mathrm{~g}$ ) and compound 12 (37 mmol, $9 \mathrm{~g}$ ) in acetic acid was stirred at $80^{\circ} \mathrm{C}$ for $2 \mathrm{~h}$. Solvent was removed under reduced pressure, and the residue was purified with flash column chromatography on silica gel to afford compound 8 ( $5.1 \mathrm{~g}, 52 \%$ yield). Yellow solid, $\mathrm{R}_{\mathrm{f}}: 0.4$ $($ EtOAc/Petroleum ether $=1: 1)$, m.p.: $177-179{ }^{\circ} \mathrm{C} .{ }^{1} \mathrm{H}$ NMR $(400 \mathrm{MHz}$, DMSO-d6) $\delta 8.29(\mathrm{~d}, J=6.1 \mathrm{~Hz}$, $1 \mathrm{H}), 7.32(\mathrm{~d}, J=6.2 \mathrm{~Hz}, 1 \mathrm{H}), 3.72-3.64(\mathrm{~m}, 4 \mathrm{H}), 3.35-3.29(\mathrm{~m}, 4 \mathrm{H}) .{ }^{13} \mathrm{C}$ NMR $(101 \mathrm{MHz}$, DMSO-d6) $\delta 151.5,150.2,149.8,130.5,113.4,109.4,65.5,50.2$. HRMS (ESI): $\mathrm{m} / \mathrm{z}\left(\mathrm{M}+\mathrm{H}^{+}\right)$calcd for $\mathrm{C}_{10} \mathrm{H}_{11} \mathrm{~N}_{4} \mathrm{O}_{3} \mathrm{~S}$, 267.0546, found: 267.0541. IR (ATR): 3109, 2985, 2861, 1585, 1504, 1312, 1209, 1108, 961, $822 \mathrm{~cm}^{-1}$.

\subsubsection{Synthesis of 7-morpholinothiazolo[5,4-b]pyridin-2-amine (14)}

A mixture of Fe powder (75.2 mmol, $4.2 \mathrm{~g}$ ) and compound 13 (18.8 mmol, $5 \mathrm{~g})$ in acetic acid was stirred at $60^{\circ} \mathrm{C}$ for $2 \mathrm{~h}$ and filtered. The filtrate was concentrated under reduced pressure, and the residue was purified with flash column chromatography on silica gel to afford compound $\mathbf{9}(2.44 \mathrm{~g}$, $55 \%$ yield). Colorless solid, $\mathrm{R}_{\mathrm{f}}: 0.4(\mathrm{DCM} / \mathrm{MeOH}=20: 1)$. m.p.: $230-232{ }^{\circ} \mathrm{C} .{ }^{1} \mathrm{H} \mathrm{NMR}(400 \mathrm{MHz}$, DMSO-d6) $\delta 7.89(\mathrm{~d}, J=5.5 \mathrm{~Hz}, 1 \mathrm{H}), 7.55(\mathrm{~s}, 2 \mathrm{H}), 6.65(\mathrm{~d}, J=5.6 \mathrm{~Hz}, 1 \mathrm{H}), 3.78-3.70(\mathrm{~m}, 4 \mathrm{H}), 3.56-3.50$ (m, 4H). ${ }^{13} \mathrm{C}$ NMR (101 MHz, DMSO-d6) $\delta$ 162.1, 155.6, 146.0, 143.0, 135.3, 107.0, 66.0, 48.3. HRMS (ESI): $m / z\left(\mathrm{M}+\mathrm{H}^{+}\right)$calcd for $\mathrm{C}_{10} \mathrm{H}_{13} \mathrm{~N}_{4} \mathrm{OS}, 237.0805$, found: 237.0807. IR (ATR): 3346, 3121, 2961, 1659, 1558, $1297,1113,973,799,700 \mathrm{~cm}^{-1}$.

\subsubsection{Synthesis of 4-(2-bromothiazolo[5,4-b]pyridin-7-yl)morpholine (15)}

A mixture of $\mathrm{CuBr}_{2}(15 \mathrm{mmol}, 3.4 \mathrm{~g})$, tert-butyl nitrite ( $\left.20 \mathrm{mmol}, 2.0 \mathrm{~g}\right)$ and compound 14 (10 mmol, $2.4 \mathrm{~g})$ in acetonitrile $(20 \mathrm{~mL})$ was stirred at room temperature for $2 \mathrm{~h}$. Water $(20 \mathrm{~mL})$ was added, and aqueous layer was extracted by $\mathrm{DCM}(20 \mathrm{~mL} \times 2)$. The organic layer was combined and washed with brine and dried over anhydrous $\mathrm{MgSO}_{4}$. $\mathrm{DCM}$ was then removed under reduced pressure and the residue was purified with flash column chromatography on silica gel to afford the compound $\mathbf{1 0}$ (1.2 g, 40\% yield. Colorless solid, $\mathrm{R}_{\mathrm{f}}: 0.4$ (EtOAc/Petroleum ether $\left.=1: 1\right)$. m.p.: $168-170{ }^{\circ} \mathrm{C} .{ }^{1} \mathrm{H}$ NMR $(400 \mathrm{MHz}, \mathrm{DMSO}-d 6) \delta 8.23(\mathrm{~d}, J=5.8 \mathrm{~Hz}, 1 \mathrm{H}), 6.88(\mathrm{~d}, J=5.9 \mathrm{~Hz}, 1 \mathrm{H}), 3.82-3.74(\mathrm{~m}, 8 \mathrm{H}) .{ }^{13} \mathrm{C} \mathrm{NMR}$ (101 MHz, DMSO-d6) $\delta 161.1,148.8,148.2,134.2,132.7,106.9,65.8,48.3$. HRMS (ESI): $m / z\left(\mathrm{M}+\mathrm{H}^{+}\right.$) calcd for $\mathrm{C}_{10} \mathrm{H}_{11} \mathrm{BrN}_{3} \mathrm{OS}$, 299.9801, found: 299.9802. IR (ATR): 3014, 2948, 2865, 1568, 1463, 1259, 1120, $1008,956,803,669 \mathrm{~cm}^{-1}$.

\subsubsection{General Procedure for the Synthesis of Aryl Borates 18a-18f}

Sulfonyl chloride (1.2 equiv.) was added to a mixture of compound 16 ( $20 \mathrm{mmol})$, DMAP ( $2 \mathrm{mmol})$ and pyridine $(30 \mathrm{mmol})$ in the DCM $(50 \mathrm{~mL})$, and the reaction; mixture was stirred at room temperature overnight. Solvent was then removed under reduced pressure and the residue was purified with flash column chromatography on silica gel to give the compound 17a-17f. KOAc ( $25 \mathrm{mmol})$ was added to a mixture of $\mathrm{PdCl}_{2}$ (dppf) (0.63 $\left.\mathrm{mmol}\right)$, compound $\mathbf{1 7 a}-\mathbf{1 7 f}(12.5 \mathrm{mmol})$ and bis(pinacolato)diboron $(15 \mathrm{mmol})$ in 1,4-dioxane $(25 \mathrm{~mL})$, and the reaction mixture was stirred at $100{ }^{\circ} \mathrm{C}$ for $5 \mathrm{~h}$. Solvent was removed under reduced pressure, and DCM $(20 \mathrm{~mL})$ was added. Then the mixture was filtered, and the filtrate was concentrated under reduced pressure and the residue was purified with flash column chromatography on silica gel to give the compound 18a-18f. 
2,4-Difluoro-N-(2-methoxy-5-(4,4,5,5-tetramethyl-1,3,2-dioxaborolan-2-yl)pyridin-3-yl)benzenesulfonamide (18a). Colorless solid (3.5 g, 66\% yield), m.p.: 163-165 ${ }^{\circ} \mathrm{C} .{ }^{1} \mathrm{H}$ NMR $(400 \mathrm{MHz}, \mathrm{DMSO}-d 6) \delta 10.18(\mathrm{~s}, 1 \mathrm{H}), 8.21$ (s, 1H), $7.72(\mathrm{~s}, 1 \mathrm{H}), 7.71-7.65(\mathrm{~m}, 1 \mathrm{H}), 7.60-7.52(\mathrm{~m}, 1 \mathrm{H}), 7.22-7.16(\mathrm{~m}, 1 \mathrm{H}), 3.62(\mathrm{~s}, 3 \mathrm{H}), 1.29(\mathrm{~s}, 12 \mathrm{H})$. ${ }^{13} \mathrm{C}$ NMR (101 MHz, DMSO-d6). $\delta 165.0(\mathrm{dd}, J=255.2,12.1 \mathrm{~Hz}), 160.2,159.3(\mathrm{dd}, J=258.6,14.1 \mathrm{~Hz})$, $150.9,141.0,131.8(\mathrm{~d}, J=11.1 \mathrm{~Hz}), 125.0(\mathrm{dd}, J=15.2,4.0 \mathrm{~Hz}), 119.2,116.7,111.8(\mathrm{dd}, J=22.2,4.0 \mathrm{~Hz})$, $105.7(\mathrm{t}, J=26.3 \mathrm{~Hz}), 84.0,53.4,24.6$. HRMS (ESI): $\mathrm{m} / \mathrm{z}\left(\mathrm{M}+\mathrm{H}^{+}\right)$calcd for $\mathrm{C}_{18} \mathrm{H}_{22} \mathrm{BF}_{2} \mathrm{~N}_{2} \mathrm{O}_{5} \mathrm{~S}, 427.1305$, found: 427.1314. IR (ATR): 3264, 2989, 2883, 1600, 1406, 1340, 1139, 1019, 969, 850, $670 \mathrm{~cm}^{-1}$.

2-Chloro-4-fluoro-N-(2-methoxy-5-(4,4,5,5-tetramethyl-1,3,2-dioxaborolan-2-yl)pyridin-3-yl)benzenesulfonamide (18b). Colorless solid (3.4 g, 61\% yield), m.p.: 138-140 ${ }^{\circ} \mathrm{C} .{ }^{1} \mathrm{H}$ NMR (400 MHz, DMSO-d6) $\delta 10.07$ $(\mathrm{s}, 1 \mathrm{H}), 8.18(\mathrm{~d}, J=1.6 \mathrm{~Hz}, 1 \mathrm{H}), 7.85(\mathrm{dd}, J=8.8,6.0 \mathrm{~Hz}, 1 \mathrm{H}), 7.73(\mathrm{dd}, J=8.7,2.5 \mathrm{~Hz}, 1 \mathrm{H}), 7.68$ $(\mathrm{d}, J=1.5 \mathrm{~Hz}, 1 \mathrm{H}), 7.34(\mathrm{td}, J=8.5,2.5 \mathrm{~Hz}, 1 \mathrm{H}), 3.63(\mathrm{~s}, 3 \mathrm{H}), 1.28(\mathrm{~s}, 12 \mathrm{H}) .{ }^{13} \mathrm{C} \mathrm{NMR}(101 \mathrm{MHz}$, DMSO-d6) $\delta 163.8(\mathrm{~d}, J=255.9 \mathrm{~Hz}), 159.9,150.6,140.1,134.5(\mathrm{~d}, J=3.4 \mathrm{~Hz}), 133.2(\mathrm{~d}, J=11.6 \mathrm{~Hz}), 132.9$ $(\mathrm{d}, J=10.3 \mathrm{~Hz}), 119.5,119.2(\mathrm{~d}, J=24.7 \mathrm{~Hz}), 117.1,114.5(\mathrm{~d}, J=21.9 \mathrm{~Hz}), 84.0,53.4,24.6$. HRMS (ESI): $m / z\left(\mathrm{M}+\mathrm{H}^{+}\right)$calcd for $\mathrm{C}_{18} \mathrm{H}_{22} \mathrm{BClFN}_{2} \mathrm{O}_{5} \mathrm{~S}, 443.1010$, found: 443.1001. IR (ATR): 3288, 3103, 2981, 1586, $1341,1154,908,850,679 \mathrm{~cm}^{-1}$.

5-Chloro-N-(2-methoxy-5-(4,4,5,5-tetramethyl-1,3,2-dioxaborolan-2-yl)pyridin-3-yl)thiophene-2-sulfonamide (18c). Colorless solid (3.6 g, 67\% yield), m.p.: 156-158 ${ }^{\circ} \mathrm{C} .{ }^{1} \mathrm{H}$ NMR (400 MHz, DMSO-d6) $\delta 10.21(\mathrm{~s}, 1 \mathrm{H})$, $8.24(\mathrm{~s}, 1 \mathrm{H}), 7.73(\mathrm{~s}, 1 \mathrm{H}), 7.29(\mathrm{~d}, J=4.1 \mathrm{~Hz}, 1 \mathrm{H}), 7.20(\mathrm{~d}, J=4.1 \mathrm{~Hz}, 1 \mathrm{H}), 3.71(\mathrm{~s}, 3 \mathrm{H}), 1.29(\mathrm{~s}, 12 \mathrm{H})$. ${ }^{13} \mathrm{C}$ NMR (101 MHz, DMSO-d6) $\delta 160.4,151.2,140.4,139.4,135.5,132.5,128.2,119.9,117.2,84.5,54.1$, 25.1. HRMS (ESI): $m / z\left(\mathrm{M}+\mathrm{H}^{+}\right)$calcd for $\mathrm{C}_{16} \mathrm{H}_{21} \mathrm{BClN}_{2} \mathrm{O}_{5} \mathrm{~S}_{2}, 431.0668$, found: 431.0680 . IR (ATR): $3251,3088,2924,1599,1397,1344,1253,1153,992,852,679 \mathrm{~cm}^{-1}$.

N-(2-Methoxy-5-(4,4,5,5-tetramethyl-1,3,2-dioxaborolan-2-yl)pyridin-3-yl)methanesulfonamide (18d). Colorless solid (2.4 g, 58\% yield), ${ }^{1} \mathrm{H}$ NMR (400 MHz, DMSO-d6) $\delta 9.23(\mathrm{~s}, 1 \mathrm{H}), 8.20(\mathrm{~s}, 1 \mathrm{H}), 7.77(\mathrm{~s}, 1 \mathrm{H})$, $3.93(\mathrm{~d}, J=3.6 \mathrm{~Hz}, 3 \mathrm{H}), 3.01(\mathrm{~s}, 3 \mathrm{H}), 1.29(\mathrm{~s}, 12 \mathrm{H}) .{ }^{13} \mathrm{C}$ NMR $(101 \mathrm{MHz}, \mathrm{DMSO}-d 6) \delta$ 158.8, 149.24, 137.0, 121.0, 116.6, 83.9, 73.5, 53.8, 40.4, 24.9, 24.6. HRMS (ESI): $m / z\left(\mathrm{M}+\mathrm{H}^{+}\right)$calcd for $\mathrm{C}_{13} \mathrm{H}_{22} \mathrm{BN}_{2} \mathrm{O}_{5} \mathrm{~S}$, 329.1337, found: 329.1318. IR (ATR): 3263, 2979, 1603, 1497, 1392, 1256, 1138, 970, 850, 771, $670 \mathrm{~cm}^{-1}$.

2,4-Difluoro-N-(2-methoxy-5-(4,4,5,5-tetramethyl-1,3,2-dioxaborolan-2-yl)phenyl)benzenesulfonamide (18e). Colorless solid (3.3 g, 62\% yield), m.p.: 150-152 ${ }^{\circ} \mathrm{C} .{ }^{1} \mathrm{H}$ NMR (400 MHz, DMSO-d6) $\delta 9.83(\mathrm{~s}, 1 \mathrm{H})$, $7.64(\mathrm{dd}, J=14.9,8.4 \mathrm{~Hz}, 1 \mathrm{H}), 7.57-7.47(\mathrm{~m}, 3 \mathrm{H}), 7.16(\mathrm{td}, J=8.6,2.1 \mathrm{~Hz}, 1 \mathrm{H}), 6.92(\mathrm{~d}, J=8.2 \mathrm{~Hz}$, 1H), 3.49 (s, 3H), 1.29 (s, 12H). ${ }^{13} \mathrm{C}$ NMR (101 MHz, DMSO-d6) $\delta 164.8$ (dd, J = 254.5, $\left.12.1 \mathrm{~Hz}\right), 159.4$ $(\mathrm{dd}, J=258.6,13.1 \mathrm{~Hz}), 156.5,134.8,134.5,131.8(\mathrm{~d}, J=11.1 \mathrm{~Hz}), 125.3(\mathrm{dd}, J=14.1,4.0 \mathrm{~Hz}), 123.7,120.1$, $111.4(\mathrm{dd}, J=22.2,3.0 \mathrm{~Hz}), 111.3,105.5(\mathrm{t}, J=30.3 \mathrm{~Hz}), 83.6,55.2,24.7$. HRMS (ESI): $m / z\left(\mathrm{M}+\mathrm{H}^{+}\right)$calcd for $\mathrm{C}_{19} \mathrm{H}_{23} \mathrm{BF}_{2} \mathrm{NO}_{5} \mathrm{~S}, 426.1353$, found: 426.1369. IR (ATR): 3300, 2925, 2850, 1601, 1340, 1258 1167, 1129, $850,669 \mathrm{~cm}^{-1}$.

\subsubsection{General Procedure for the Synthesis of Target Compounds $19 a-19 f$}

$\mathrm{K}_{2} \mathrm{CO}_{3}$ aqueous solution $(2 \mathrm{~N}, 0.39 \mathrm{~mL})$ was added to a mixture of $\mathrm{PdCl}_{2}(\mathrm{dppf})(0.03 \mathrm{mmol})$, compound $15(0.26 \mathrm{mmol})$ and Aryl borate $18(0.31 \mathrm{mmol})$ in 1,4-dioxane $(2.5 \mathrm{~mL})$, and the reaction mixture was stirred at $100{ }^{\circ} \mathrm{C}$ for $5 \mathrm{~h}$. Solvent was removed under reduced pressure, and DCM (20 mL) was added. Then the mixture was filtered, and the filtrate was concentrated under reduced pressure and the residue was purified with flash column chromatography on silica gel to give compound 19. ${ }^{1} \mathrm{H}$ NMR and ${ }^{13}$ CNMR spectra of the compounds 19a-19f, and the HPLC spectra of compound 19a can be found in the Supplementary Materials.

2,4-Difluoro-N-(2-methoxy-5-(7-morpholinothiazolo[5,4-b]pyridin-2-yl)pyridin-3-yl)benzenesulfonamide (19a). Colorless solid (103 mg, 76\% yield), $\mathrm{R}_{\mathrm{f}}: 0.5$ (DCM/MeOH = 30:1), m.p.: $175-177{ }^{\circ} \mathrm{C} .{ }^{1} \mathrm{H} \mathrm{NMR}(400 \mathrm{MHz}$, DMSO-d6) $\delta 10.52(\mathrm{~s}, 1 \mathrm{H}), 8.62(\mathrm{~d}, J=2.1 \mathrm{~Hz}, 1 \mathrm{H}), 8.21(\mathrm{~d}, J=5.7 \mathrm{~Hz}, 1 \mathrm{H}), 8.11(\mathrm{~d}, J=2.1 \mathrm{~Hz}, 1 \mathrm{H})$, $7.80(\mathrm{dd}, J=14.9,8.5 \mathrm{~Hz}, 1 \mathrm{H}), 7.64-7.56(\mathrm{~m}, 1 \mathrm{H}), 7.25(\mathrm{td}, J=8.6,2.1 \mathrm{~Hz}, 1 \mathrm{H}), 6.86(\mathrm{~d}, J=5.7 \mathrm{~Hz}, 1 \mathrm{H})$, 3.88-3.78 (m, 8H), 3.75 (s, 3H). ${ }^{13} \mathrm{C}$ NMR (101 MHz, DMSO-d6) $\delta 165.1(\mathrm{dd}, J=255.5 \mathrm{~Hz}, 12.1 \mathrm{~Hz}), 159.4$, 
$159.3(\mathrm{dd}, J=258.6 \mathrm{~Hz}, 14.1 \mathrm{~Hz}), 158.9,157.2,149.3,147.9,142.7,135.2,131.8(\mathrm{~d}, J=11.1 \mathrm{~Hz}), 131.2,124.8$ $(\mathrm{dd}, J=14.1 \mathrm{~Hz}, 3.0 \mathrm{~Hz}), 123.4,120.5,112.1(\mathrm{dd}, J=22.2 \mathrm{~Hz}, 3.0 \mathrm{~Hz}), 106.4,105.9(\mathrm{t}, J=26.3 \mathrm{~Hz}), 65.9$, 54.0, 48.5. HRMS (ESI): $\mathrm{m} / \mathrm{z}\left(\mathrm{M}+\mathrm{H}^{+}\right)$calcd for $\mathrm{C}_{22} \mathrm{H}_{20} \mathrm{~F}_{2} \mathrm{~N}_{5} \mathrm{O}_{4} \mathrm{~S}_{2}, 520.0919$, found: 520.0909. IR (ATR): $3183,2922,2854,1602,1423,1261,1149,971,864,715,668 \mathrm{~cm}^{-1}$.

2-Chloro-4-fluoro-N-(2-methoxy-5-(7-morpholinothiazolo[5,4-b]pyridin-2-yl)pyridin-3-yl)benzenesulfonamide (19b). Colorless solid (102 mg, 73\% yield), $\mathrm{R}_{\mathrm{f}}: 0.5(\mathrm{DCM} / \mathrm{MeOH}=30: 1)$, m.p.: $188-190{ }^{\circ} \mathrm{C} .{ }^{1} \mathrm{H} \mathrm{NMR}$ $(400 \mathrm{MHz}$, DMSO-d6) $\delta 10.45(\mathrm{~s}, 1 \mathrm{H}), 8.60(\mathrm{~d}, J=2.1 \mathrm{~Hz}, 1 \mathrm{H}), 8.20(\mathrm{~d}, J=5.7 \mathrm{~Hz}, 1 \mathrm{H}), 8.07(\mathrm{~d}, J=2.1 \mathrm{~Hz}$, $1 \mathrm{H}), 7.97(\mathrm{dd}, J=8.9,5.9 \mathrm{~Hz}, 1 \mathrm{H}), 7.77(\mathrm{dd}, J=8.7,2.4 \mathrm{~Hz}, 1 \mathrm{H}), 7.40(\mathrm{td}, J=8.5,2.5 \mathrm{~Hz}, 1 \mathrm{H})$, $6.86(\mathrm{~d}, J=5.7 \mathrm{~Hz}, 1 \mathrm{H}), 3.88-3.78(\mathrm{~m}, 8 \mathrm{H}), 3.77(\mathrm{~s}, 3 \mathrm{H}) .{ }^{13} \mathrm{C}$ NMR (101 MHz, DMSO-d6) $\delta 163.9$ $(\mathrm{d}, J=255.5 \mathrm{~Hz}), 159.3,158.6,157.2,149.3,148.0,142.4,135.2,134.3(\mathrm{~d}, J=3.0 \mathrm{~Hz}), 133.1(\mathrm{~d}, J=6.1 \mathrm{~Hz})$, $133.0(\mathrm{~d}, J=4.0 \mathrm{~Hz}), 130.3,123.3,120.9,119.4(\mathrm{~d}, J=26.3 \mathrm{~Hz}), 114.7(\mathrm{~d}, J=22.2 \mathrm{~Hz}), 106.4,65.9,54.0$, 48.5. HRMS (ESI): $\mathrm{m} / z\left(\mathrm{M}+\mathrm{H}^{+}\right)$calcd for $\mathrm{C}_{22} \mathrm{H}_{20} \mathrm{ClFN}_{5} \mathrm{O}_{4} \mathrm{~S}_{2}, 536.0624$, found: 536.0612 . IR (ATR): $3376,3008,2921,1568,1471,1341,1253,1157,977,803,661 \mathrm{~cm}^{-1}$.

5-Chloro-N-(2-methoxy-5-(7-morpholinothiazolo[5,4-b]pyridin-2-yl)pyridin-3-yl)thiophene-2-sulfonamide (19c). Colorless solid (95 mg, 70\% yield), $\mathrm{R}_{\mathrm{f}}: 0.5(\mathrm{DCM} / \mathrm{MeOH}=30: 1)$, m.p.: $116-118{ }^{\circ} \mathrm{C} .{ }^{1} \mathrm{H}$ NMR $(400 \mathrm{MHz}$, DMSO-d6) $\delta 10.56(\mathrm{~s}, 1 \mathrm{H}), 8.63(\mathrm{~d}, J=1.9 \mathrm{~Hz}, 1 \mathrm{H}), 8.21(\mathrm{~d}, J=5.6 \mathrm{~Hz}, 1 \mathrm{H}), 8.14(\mathrm{~d}, J=2.0 \mathrm{~Hz}, 1 \mathrm{H}), 7.44$ $(\mathrm{d}, J=4.1 \mathrm{~Hz}, 1 \mathrm{H}), 7.25(\mathrm{~d}, J=4.1 \mathrm{~Hz}, 1 \mathrm{H}), 6.87(\mathrm{~d}, J=5.7 \mathrm{~Hz}, 1 \mathrm{H}), 3.91-3.80(\mathrm{~m}, 11 \mathrm{H}) .{ }^{13} \mathrm{C}$ NMR $(101$ MHz, DMSO-d6) $\delta$ 159.3, 158.5, 157.4, 149.3, 148.0, 142.3, 138.8, 135.4, 135.3, 132.4, 129.4, 128.1, 123.4, 121.0, 106.5, 66.0, 54.2, 48.5. HRMS (ESI): $\mathrm{m} / \mathrm{z}\left(\mathrm{M}+\mathrm{H}^{+}\right)$calcd for $\mathrm{C}_{20} \mathrm{H}_{20} \mathrm{ClN}_{5} \mathrm{O}_{4} \mathrm{~S}_{3}, 524.0282$, found: 524.0285. IR (ATR): 33503015 2921, 1566, 1406, 1341, 1256, 1149, 976, 802, $676 \mathrm{~cm}^{-1}$.

N-(2-Methoxy-5-(7-morpholinothiazolo[5,4-b]pyridin-2-yl)pyridin-3-yl)methanesulfonamide (19d). Colorless solid (73 mg, 67\% yield), $\mathrm{R}_{\mathrm{f}}: 0.5(\mathrm{DCM} / \mathrm{MeOH}=30: 1)$,m.p.: $230-232{ }^{\circ} \mathrm{C} .{ }^{1} \mathrm{H} \mathrm{NMR}(400 \mathrm{MHz}$, DMSO-d6) $\delta 9.56(\mathrm{~s}, 1 \mathrm{H}), 8.63(\mathrm{~d}, J=2.2 \mathrm{~Hz}, 1 \mathrm{H}), 8.24-8.20(\mathrm{~m}, 2 \mathrm{H}), 6.89(\mathrm{~d}, J=5.7 \mathrm{~Hz}, 1 \mathrm{H}), 4.02(\mathrm{~s}, 3 \mathrm{H})$, 3.92-3.80 (m, 8H), 3.13 (s, 3H). ${ }^{13} \mathrm{C}$ NMR (101 MHz, DMSO-d6) 8 159.4, 157.7, 157.6, 149.3, 148.0, 140.9, $135.3,128.1,123.5,122.4,106.5,65.9,54.3,48.5,40.6$. HRMS (ESI): $\mathrm{m} / z\left(\mathrm{M}+\mathrm{H}^{+}\right)$calcd for $\mathrm{C}_{17} \mathrm{H}_{20} \mathrm{~N}_{5} \mathrm{O}_{4} \mathrm{~S}_{2}$, 422.0951, found: 422.0944. IR (ATR): 3215, 2998, 2882, 1585, 1464, 1393, 1287, 1137, 1003, 841, 771, $684 \mathrm{~cm}^{-1}$.

2,4-Difluoro-N-(2-methoxy-5-(7-morpholinothiazolo[5,4-b]pyridin-2-yl)phenyl)benzenesulfonamide (19e). Colorless solid (101 mg, 75\% yield), $\mathrm{R}_{\mathrm{f}}: 0.5(\mathrm{DCM} / \mathrm{MeOH}=30: 1)$, m.p.: $195-197{ }^{\circ} \mathrm{C} .{ }^{1} \mathrm{H}$ NMR $(400 \mathrm{MHz}, \mathrm{DMSO}-d 6) \delta 10.15(\mathrm{~s}, 1 \mathrm{H}), 8.19(\mathrm{~d}, J=5.6 \mathrm{~Hz}, 1 \mathrm{H}), 7.84-7.80(\mathrm{~m}, 2 \mathrm{H}), 7.74(\mathrm{dd}, J=14.9,8.5$ $\mathrm{Hz}, 1 \mathrm{H}), 7.61-7.54(\mathrm{~m}, 1 \mathrm{H}), 7.21(\mathrm{t}, J=7.5 \mathrm{~Hz}, 1 \mathrm{H}), 7.11(\mathrm{~d}, J=8.4 \mathrm{~Hz}, 1 \mathrm{H}), 6.86(\mathrm{~d}, J=5.7 \mathrm{~Hz}, 1 \mathrm{H})$, 3.89-3.79 (m, 8H), $3.62(\mathrm{~s}, 3 \mathrm{H}) .{ }^{13} \mathrm{C}$ NMR (101 MHz, DMSO-d6) $\delta 165.0(\mathrm{dd}, J=253.5 \mathrm{~Hz}, 14.1 \mathrm{~Hz})$, 160.0, 159.3, $159.2(\mathrm{dd}, J=258.6 \mathrm{~Hz}, 15.2 \mathrm{~Hz}), 155.6,149.3,147.7,135.6,131.8(\mathrm{~d}, J=1.1 \mathrm{~Hz}), 126.6$, $125.5,125.3,125.1(\mathrm{dd}, J=14.1 \mathrm{~Hz}, 4.0 \mathrm{~Hz}), 124.7,112.5,111.8(\mathrm{dd}, J=23.2 \mathrm{~Hz}, 4.0 \mathrm{~Hz}), 106.5,105.8$ $(\mathrm{t}, J=26.3 \mathrm{~Hz}), 65.9,55.8,48.5$. HRMS (ESI): $\mathrm{m} / \mathrm{z}\left(\mathrm{M}+\mathrm{H}^{+}\right)$calcd for $\mathrm{C}_{23} \mathrm{H}_{21} \mathrm{~F}_{2} \mathrm{~N}_{4} \mathrm{O}_{4} \mathrm{~S}_{2}, 519.0967$, found: 519.0970. IR (ATR): 3268, 2922, 2844, 1567, 1426, 1336, 1272, 1122, 969, 816, $670 \mathrm{~cm}^{-1}$.

2,4-Difluoro-N-(5-(7-morpholinothiazolo[5,4-b]pyridin-2-yl)pyridin-3-yl)benzenesulfonamide (19f). Colorless solid ( $57 \mathrm{mg}$, 45\% yield), $\mathrm{R}_{\mathrm{f}}: 0.3(\mathrm{DCM} / \mathrm{MeOH}=30: 1)$. m.p.: $99-101{ }^{\circ} \mathrm{C} .{ }^{1} \mathrm{H} \mathrm{NMR}(400 \mathrm{MHz}$, DMSO-d6) $\delta 11.35(\mathrm{~s}, 1 \mathrm{H}), 8.87(\mathrm{~s}, 1 \mathrm{H}), 8.47(\mathrm{~d}, J=2.0 \mathrm{~Hz}, 1 \mathrm{H}), 8.23(\mathrm{~d}, J=5.7 \mathrm{~Hz}, 1 \mathrm{H}), 8.08(\mathrm{~s}, 1 \mathrm{H}), 8.00$ $(\mathrm{dd}, J=14.8,8.5 \mathrm{~Hz}, 1 \mathrm{H}), 7.61-7.54(\mathrm{~m}, 1 \mathrm{H}), 7.32(\mathrm{t}, J=8.4 \mathrm{~Hz}, 1 \mathrm{H}), 6.89(\mathrm{~d}, J=5.7 \mathrm{~Hz}, 1 \mathrm{H}), 3.92-3.80$ $(\mathrm{m}, 8 \mathrm{H}) .{ }^{13} \mathrm{C}$ NMR $(101 \mathrm{MHz}, \mathrm{DMSO}-d 6) \delta 165.5(\mathrm{dd}, J=256.5,12.1 \mathrm{~Hz}), 159.6,159.0(\mathrm{dd}, J=258.6$, $14.1 \mathrm{~Hz}), 156.9,149.6,148.5,142.9,142.5,135.3,134.6,132.4(\mathrm{~d}, J=10.1 \mathrm{~Hz}), 129.1,123.4(\mathrm{dd}, J=14.1$, $3.0 \mathrm{~Hz}), 123.2,112.7(\mathrm{dd}, J=22.2,4.0 \mathrm{~Hz}), 106 .(\mathrm{t}, J=25.3 \mathrm{~Hz}), 65.9,48.5$. HRMS (ESI): $m / z\left(\mathrm{M}+\mathrm{H}^{+}\right)$ calcd for $\mathrm{C}_{21} \mathrm{H}_{18} \mathrm{~F}_{2} \mathrm{~N}_{5} \mathrm{O}_{3} \mathrm{~S}_{2}, 490.0814$, found: 490.0805. IR (ATR): 3000, 2928, 1557, 1476, 1425, 1343, $1266,1068,961,846,669 \mathrm{~cm}^{-1}$. 


\section{Conclusions}

In summary, we have designed and synthesized a series of 2-pyridyl, 4-morpholinyl substituted thiazolo[5,4- $b$ ]pyridines by pharmacophore splicing of methoxyl pyridine and morpholinyl heterocyclic inhibitors. These molecules were evaluated for the inhibitory activity of kinases. It was found that compound 19a exhibited nanomolar inhibitory activity against the three isoforms of $\mathrm{PI} 3 \mathrm{~K} \alpha, \mathrm{PI} 3 \mathrm{~K} \gamma$, and PI3K $\delta$. Further docking study demonstrated that compound 19a fit well into the ATP binding pocket of the PI3K $\alpha$ kinase. Overall, these novel thiazolo[5,4- $b$ ]pyridines may be potentially used as the potent PI3K inhibitors for the treatment of disease mediated by the PI3K signaling pathway after further activity evaluation in vivo and optimization of their pharmacological properties.

Supplementary Materials: ${ }^{1} \mathrm{H}$ NMR and ${ }^{13} \mathrm{CNMR}$ spectra of the compounds 19a-19f, and the HPLC spectra of compound 19a.

Author Contributions: L.X., Y.Z., J.Z., S.L., K.Z., and H.T. performed the experiments and analyzed the data; H.X. and Y.D. conceived and designed the study; all authors contributed to writing and editing of the paper. All authors have read and agreed to the published version of the manuscript.

Funding: This research was Funded by the National Natural Science Foundation of China (81703329 and 21702234), the CAMS Innovation Fund for Medical Sciences (2017-I2M-3-011 and 2019-I2M-1-005), the Drug Innovation Major Project (2018ZX09711-001-005), the Non-profit Central Research Institute Fund of Chinese Academy of Medical Sciences (2018PT35003 and 2019RC-HL-008), and the Fundamental Research Funds for the Central Universities (3332020041).

Acknowledgments: We thank Xudong Wu for his help on molecular modeling.

Conflicts of Interest: The authors declare no conflict of interest.

\section{References}

1. Balaban, A.T. Aromaticity as a Cornerstone of Heterocyclic Chemistry. Chem. Rev. 2004, 104, $2777-2812$. [CrossRef] [PubMed]

2. Taylor, R.D.; MacCoss, M.; Lawson, A.D.G. Rings in Drugs. J. Med. Chem. 2014, 57, 5845-5859. [CrossRef]

3. Mullard, A. 2012 FDA Drug Approvals. Nat. Rev. Drug Discov. 2013, 12, 87-90. [CrossRef] [PubMed]

4. Das, P.; Delost, M.D.; Qureshi, M.H.; Smith, D.T.; Njardarson, J.T. A Survey of the Structures of US FDA Approved Combination Drugs. J. Med. Chem. 2019, 62, 4265-4311. [CrossRef] [PubMed]

5. Ertl, P.; Jelfs, S.; Mühlbacher, J.; Schuffenhauer, A.; Selzer, P. Quest for the Rings. In Silico Exploration of Ring Universe To Identify Novel Bioactive Heteroaromatic Scaffolds. J. Med. Chem. 2006, 49, 4568-4573. [CrossRef] [PubMed]

6. Pitt, W.R.; Parry, D.M.; Perry, B.G.; Groom, C.R. Heteroaromatic Rings of the Future. J. Med. Chem. 2009, 52, 2952-2963. [CrossRef]

7. Gibson, S.; McGuire, R.; Rees, D.C. Principal Components Describing Biological Activities and Molecular Diversity of Heterocyclic Aromatic Ring Fragments. J. Med. Chem. 1996, 39, 4065-4072. [CrossRef]

8. Kalaria, P.N.; Karad, S.C.; Raval, D.K. A Review on Diverse Heterocyclic Compounds as the Privileged Scaffolds in Antimalarial Drug Discovery. Eur. J. Med. Chem. 2018, 5, 917-936. [CrossRef]

9. Vitaku, E.; Smith, D.T.; Njardarson, J.T. Analysis of the Structural Diversity, Substitution Patterns, and Frequency of Nitrogen Heterocycles among U.S. FDA Approved Pharmaceuticals. J. Med. Chem. 2014, 57, 10257-10274. [CrossRef]

10. Taylor, A.P.; Robinson, R.P.; Fobian, Y.M.; Blakemore, D.C.; Jones, L.H.; Fadeyi, O. Modern Advances in Heterocyclic Chemistry in Drug Discovery. Org. Biomol. Chem. 2016, 14, 6611-6637. [CrossRef]

11. Kuppast, B.; Fahmy, H. Thiazolo[4,5-d]pyrimidines as a Privileged Scaffold in Drug Discovery. Eur. J. Med. Chem. 2016, 113, 198-213. [CrossRef] [PubMed]

12. Cee, V.J.; Frohn, M.; Lanman, B.A.; Golden, J.; Muller, K.; Neira, S.; Pickrell, A.; Arnett, H.; Buys, J.; Gore, A.; et al. Discovery of AMG 369, a Thiazolo[5,4-b]pyridine Agonist of S1P1 and S1P5. ACS Med. Chem. Lett. 2011, 2, 107-112. [CrossRef] [PubMed]

13. Rao, A.U.; Palani, A.; Chen, X.; Huang, Y.; Aslanian, R.G.; West, R.E., Jr.; Williams, S.M.; Wu, R.; Hwa, J.; Sondey, C.; et al. Synthesis and Structure-activity Relationships of 2-(1,4'-bipiperidin-1'-yl)thiazolopyridine as $\mathrm{H}_{3}$ Receptor Antagonists. Bioorg. Med. Chem. Lett. 2009, 19, 6176-6180. [CrossRef] [PubMed] 
14. Kale, M.G.; Raichurkar, A.; Hameed, P.S.; Waterson, D.; McKinney, D.; Manjunatha, M.R.; Kranthi, U.; Koushik, K.; Jena, L.K.; Shinde, V.; et al. Thiazolopyridine Ureas as Novel Antitubercular Agents Acting through Inhibition of DNA Gyrase, B. J. Med. Chem. 2013, 56, 8834-8848. [CrossRef] [PubMed]

15. Xie, X.; Li, H.; Wang, J.; Mao, S.; Xin, M.; Lu, S.; Mei, Q.; Zhang, S. Synthesis and Anticancer Effects Evaluation of 1-alkyl-3-(6-(2-methoxy-3-sulfonylaminopyridin-5-yl)benzo[d]thiazol-2-yl)urea as Anticancer Agents with Low Toxicity. Bioorg. Med. Chem. 2015, 23, 6477-6485. [CrossRef]

16. Bebernitz, G.R.; Beaulieu, V.; Dale, B.A.; Deacon, R.; Duttaroy, A.; Gao, J.; Grondine, M.S.; Gupta, R.C.; Kakmak, M.; Kavana, M.; et al. Investigation of Functionally Liver Selective Glucokinase Activators for the Treatment of Type 2 Diabetes. J. Med. Chem. 2009, 52, 6142-6152. [CrossRef]

17. Liu, P.; Cheng, H.; Roberts, T.M.; Zhao, J.J. Targeting the Phosphoinositide 3-kinase Pathway in Cancer. Nat. Rev. Drug Discov. 2009, 8, 627-644. [CrossRef]

18. Katso, R.; Okkenhaug, K.; Ahmadi, K.; White, S.; Timms, J.; Waterfield, M.D. Cellular Function of Phosphoinositide 3-kinases: Implications for Development, Homeostasis, and Cancer. Annu. Rev. Cell Dev. Biol. 2001, 17, 615-675. [CrossRef]

19. Fan, Q.W.; Knight, Z.A.; Goldenberg, D.D.; Yu, W.; Mostov, K.E.; Stokoe, D.; Shokat, K.M.; Weiss, W.A. A Dual PI3 Kinase/mTOR Inhibitor Reveals Emergent Efficacy in Glioma. Cancer Cell 2006, 9, 341-349. [CrossRef]

20. Burger, M.T.; Pecchi, S.; Wagman, A.; Ni, Z.J.; Knapp, M.; Hendrickson, T.; Atallah, G.; Pfister, K.; Zhang, Y.; Bartulis, S.; et al. Identification of NVP-BKM120 as a Potent, Selective, Orally Bioavailable Class I PI3 Kinase Inhibitor for Treating Cancer. ACS Med. Chem. Lett. 2011, 2, 774-779. [CrossRef]

21. Sutherlin, D.P.; Bao, L.; Berry, M.; Castanedo, G.; Chuckowree, I.; Dotson, J.; Folks, A.; Friedman, L.; Goldsmith, R.; Gunzner, J.; et al. Discovery of a Potent, Selective, and Orally Available Class I Phosphatidylinositol 3-kinase (PI3K)/mammalian Target of Rapamycin (mTOR) Kinase Inhibitor (GDC-0980) for the Treatment of Cancer. J. Med. Chem. 2011, 54, 7579-7587. [CrossRef]

22. Cheng, H.M.; Bagrodia, S.; Bailey, S.; Edwards, M.; Hoffman, J.; Hu, Q.Y.; Kania, R.; Knighton, D.R.; Marx, M.A.; Ninkovic, S.; et al. Discovery of the Highly Potent PI3K/mTOR Dual Inhibitor PF-04691502 Through Structure Based Drug Design. Med. Chem. Comm. 2010, 1, 139-144. [CrossRef]

23. Knight, S.D.; Adams, N.D.; Burgess, J.L.; Chaudhari, A.M.; Darcy, M.G.; Donatelli, C.A.; Luengo, J.I.; Newlander, K.A.; Parrish, C.A.; Ridgers, L.H.; et al. Discovery of GSK2126458, a Highly Potent Inhibitor of PI3K and the Mammalian Target of Rapamycin. ACS Med. Chem. Lett. 2010, 1, 39-43. [CrossRef] [PubMed]

24. Lin, S.; Wang, C.; Ji, M.; Wu, D.; Lv, Y.; Zhang, K.; Dong, Y.; Jin, J.; Chen, J.; Zhang, J.; et al. Discovery and Optimization of 2-Amino-4-methylquinazoline Derivatives as Highly Potent Phosphatidylinositol 3-Kinase Inhibitors for Cancer Treatment. J. Med. Chem. 2018, 61, 6087-6109. [CrossRef] [PubMed]

25. McDonald, S.L.; Hendrick, C.E.; Wang, Q. Copper-Catalyzed Electrophilic Amination of Heteroarenes and Arenes by C-H Zincation. Angew. Chem. Int. Ed. 2014, 53, 4667-4670. [CrossRef] [PubMed]

Sample Availability: Samples of the compounds are available from the authors.

(C) 2020 by the authors. Licensee MDPI, Basel, Switzerland. This article is an open access article distributed under the terms and conditions of the Creative Commons Attribution (CC BY) license (http://creativecommons.org/licenses/by/4.0/). 